\title{
MODULE FOR VISUALIZATION AND ANALYSIS OF ERRORS IN MAKING THREADING TOOLS - MACHINE TAPS
}

\section{Detelina Angelova, Stefan Kartunov}

\begin{abstract}
The approach, methods and means to build a research and information system and module for visualization and analysis of errors in making threading tools - machine taps for internal threadsM4, M6, M8, M10 are presented.

Keywords: module for visualization and analysis of errors
\end{abstract}

\section{INTRODUCTION}

An object of the research with the research and information system are the mistakes received in making threading tools taps of four types: M4, M6, M8, M10, the choice of technological operations for their making and studying the quality of the tools when changing the cutting regimes in these operations.

The aim is not only to bind the structural design with the technological process in making the taps, but to create a medium for visualizing the tools reporting the errors in their making.

This objective can be achieved by using an available CAD-system AutoCAD for working drawings of tools with their main parameters, the impact of which was examined by multivariate analysis or by developing a simpler module for structural variant design.

When integrating this system and a module with the part for designing of the technological operations, it is seen which of these parameters in which technological operation are received and how they can be changed to improve the quality of the manufactured tools.

The change is illustrated by visualization of the mistakes in the process for the receipt of every size and deviation from its tolerance.

\section{PRESENTATION}

From the presented above, it follows that the flowchart of the system for visualization and analysis of errors in making threading tools must be built on a modular principle and contain the following main modules: "Module for designing," "Module for selection of technological operation" and " Module for visualization".

The information of these modules accumulated in the database allows that the system be used as an information system in the training process.

The system also has a service module that allows updating of the database and solving auxiliary functions.

The structural diagrams of the system and of the database are shown in Fig. 1 and 2. The main tables from the database are presented with rectangles, and the links between them - by arrows.

Fig. 1. Structural diagram of the system

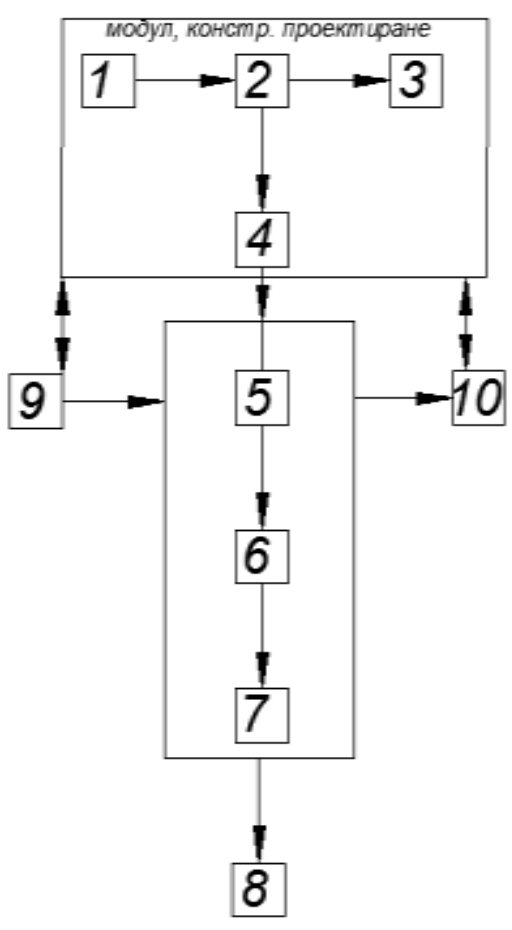


Legend:

1. Geometric design;

2. Eng. calculations and analysis;

3. Technological design;

4. Results;

5. Selection of technological operation;

6. Assessment of selected operation;

7. Results

Module for engineering design

8. Module for visualization;

9. Service functions;

10. Information system.

Fig. 2. Structural diagram of databases

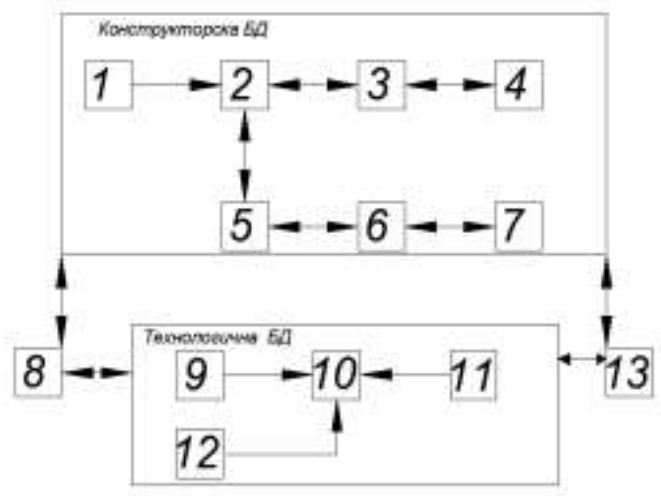

Legend:

1. Tools;

2. Ready solutions of design inform.

3. Structural modelsof design inform.;

4. Parameters of design inform;

5. Ready solutions;

6. Geometric models;

7. Parameters designed tool;

8. Administrative database;

9. Basic technological operations;

10. Ready solutions;

11. Group processes

12. Technological parameters of operation.

13. Operational database.

The main algorithm of work of the research and information system is shown in Fig. 3.

For the geometric modeling of threading tools, two basic methods for structural design are used [1]:

- Variation modeling - groups of similar tools are developed where a parametric geometric model is introduced for each of them, for example, a group of taps M8 and M10.
- There are two possibilities. If in the database already there is a geometric model that matches the designed tool, only the dimensions of the studied parameters of the tool are entered and recorded in the database as a solution, for example, groups of taps M4 and M6. If the geometry of the tool does not match any model from the database, then the closest model is selected. Adjustments to its geometry are entered, parameters are defined and it is recorded in the database as a new model in a new group of tools by entering the dimensions as a variant of values for it's parameters. This solution is recorded in the database, and the approach allows for future addition and development of the research system.

- Generative geometric modeling - If in the database there is not a model that matches the designed tool to be made, its geometry is designed, it's parameters are determined and it is recorded in the database as a new solution for the tools. In this case, an available in the market high-level CAD-system is used. Dimensions of the specific designed tool are entered as a variant of values and the data are recorded in the database. This is shown in fig. 4 .

Table 2. Drawing design tool

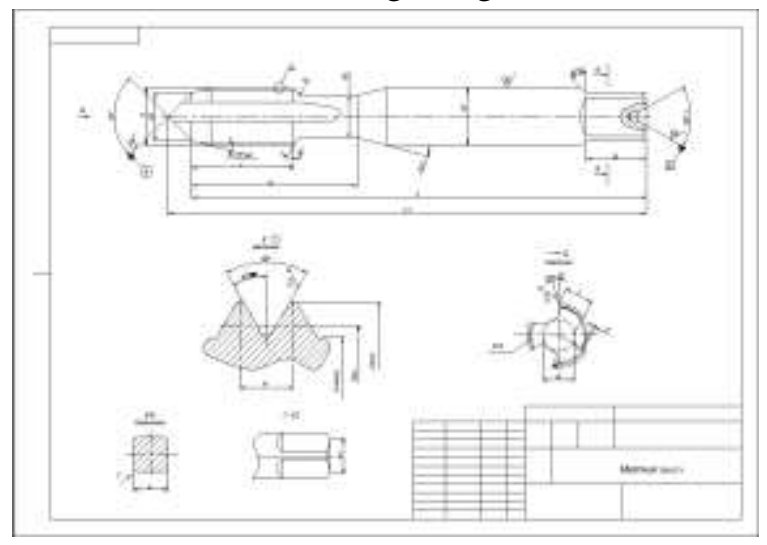


Fig . 3. Main algorithm of operation of the system

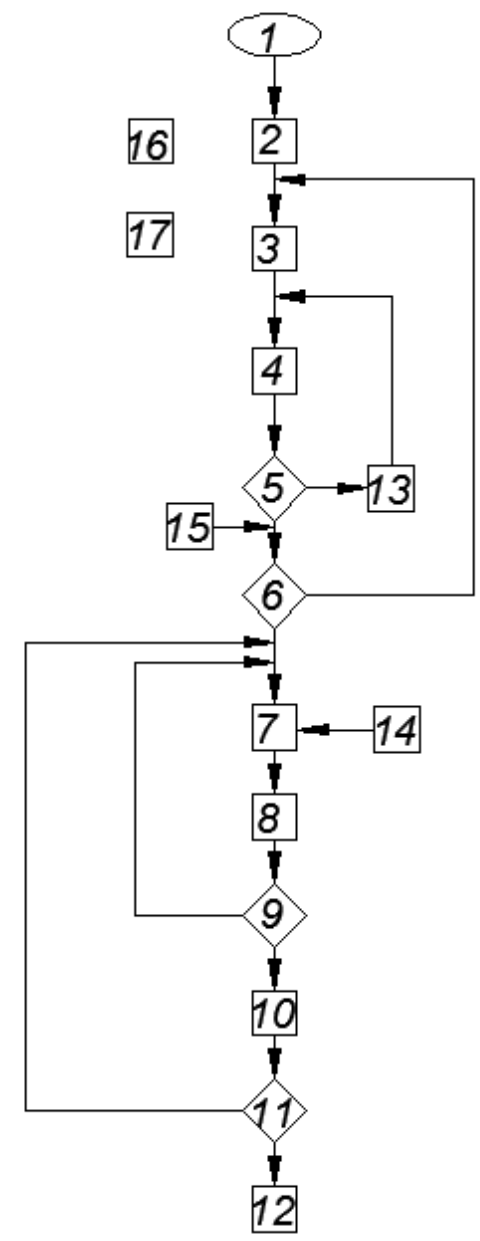

Legend:

1. Beginning;

2. Choice of instruments;

3. Geometric modeling;

4. Eng. Analysis of Tools;

5. Analysis of results;

6. Results of errors;

7. Selection of technological operation, depending on the parameters;

8. Search batch process for making technological operation;

9. Is selected group process (Yes);

10. Edit parameters;

11. Results of the evaluation of the errors;

12. Display, End.

13. Change results;

14. Technological operations;

15. Errors:

16. Tools;

17. Geometric solutions.
Fig . 4. Generative geometric modeling

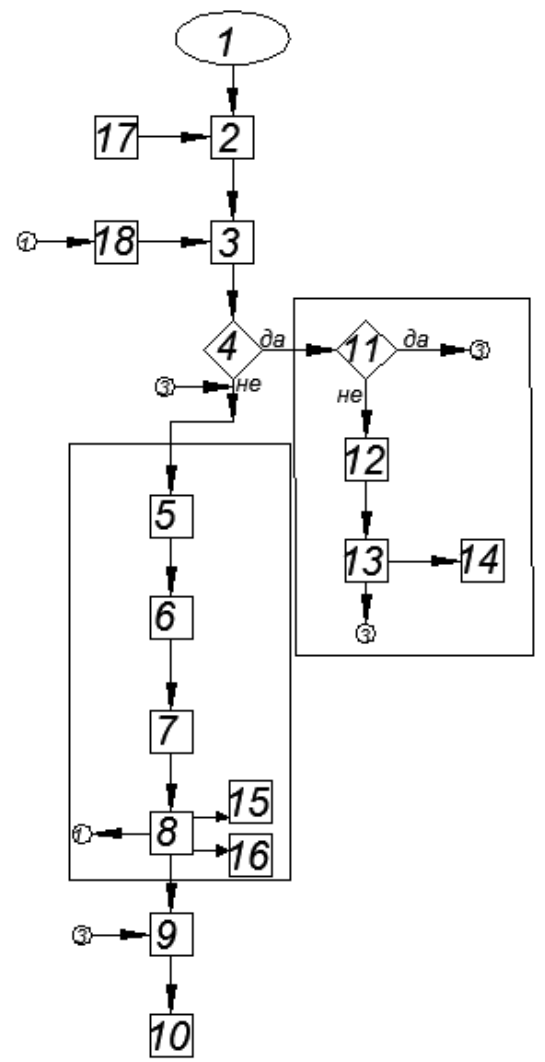

Legend:

1. Home;

2. Selection of instruments;

3. Search group with geometric solutions for design tool;

4. It found a group of geometric solutions for design tool;

5. Drawing geometric model designed tool;

6. Defining the parameters of the geometrical model of the instrument;

7. Create option values for the parameters of the technological model of the instrument;

8. Recording a geometric pattern, geometric group decisions and options to the values of the parameters for the design tool;

9. Submission of parameters designed instrument module Eng. Analysis to assess the project decision;

10. End;

11. Is there an option values for the parameters of the designed tool;

12. Create option values for parameters designed tool;

13. Recording the values of parameters in the database design tool.

14. Variants values Tool. 
When choosing a technological operation, two approaches fordesign are also used - variant and generative [1]. The basis of the variant method is the unification of the tools and processes by using group processing. In the generative method, a synthesis of the technological process is provided. Here a variant approach for design is used. For each group of tools, group technological processes with different modes of working operations are developed. Their choice is determined by the quality of the tools. The flow chart of the algorithm is shown in fig. 5

Fig . 5. Flowchart of the algorithm for technological design

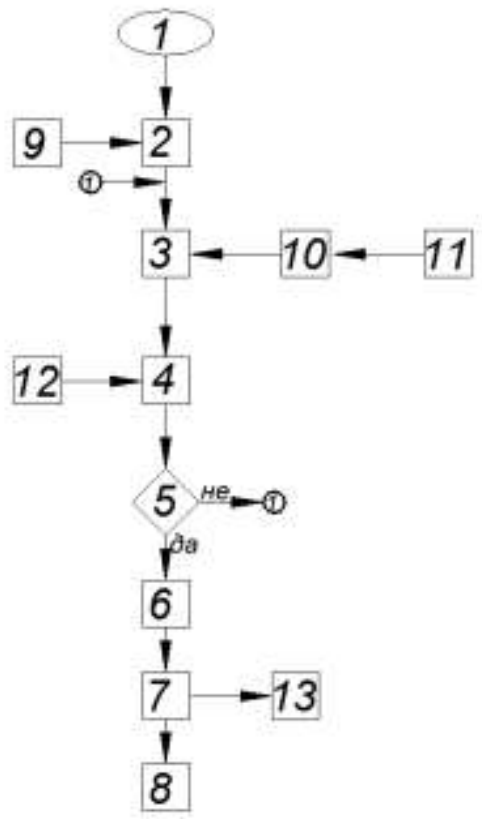

Legend:

1. Home;

2. Introduction of the parameters of structural design of the instrument;

3 . The operation selection processing depending on the geometry of the material of the instrument;

4. Search for group process on selected processing operation;

5. Is Found group TA design tool;

6. Edit the parameters of the TP Group;

7. Save the designed process in the database;

8. End;

9. Module structural design;

10. Tables of applicability;
11. Processing operations;

12. Group processes;

13. Ready solutions to the process.

The third module is visualization of errors. The flowchart of this module consists of the elements: selection from the main menu, geometric dimensions of the taps, selection of the working operation, entering an error of 4 types of errors in the dimensions of the tools and connection with AutoCAD. The submenus of the main programme for configuring the system, for the databases used and operating instructions are located in the main menu. On the visualization window it self, there are buttons for producing, data of the tap, start of AutoCAD, entering an error, close and output. On the window for producing, a technological operation is set with the option to change the cutting modes or selection of a new operation. On the window for the data of the tap, 4 dimensions must be set, studied by a multi-criteria analysis, as well - and additional ones. The launch of the PPP AutoCAD is necessary when using a generative geometric modeling. Setting of an error is done by selecting one of the errors determined as important, discussed in [3], and specific numerical values are entered. The visualization module allows to simulate the process of making taps and to receive four types of errors in it.

Fig. 6. Module preview

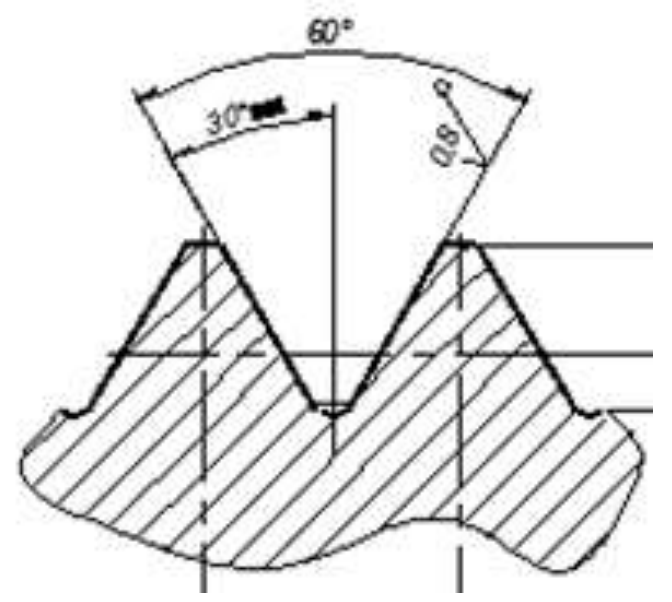

Module Visualization allows simulation the processing of taps. 
The second part allows simulation of the four types of errors in the processing of taps [4].

The databases of the system aim to collect and systematize the necessary data for the tools and machining machines, necessary for the process of making taps [2]. They may be supplemented by the user, which increases the capabilities of the modules. Administrative and operational database (DB) is needed for the operation of the system.

\section{EXPERIMENTAL RESULTS}

For the designed structural diagram from fig. 1 and the main algorithm of operation of the research and information system for visualization and analysis of errors in making threading tools, software for "Module for Structural Design", "Module for Technological Design" and "Module for Visualization" by Delphi has been developed, which is a subject of another publication. According to the designed structural diagram of the database, a design database based on a model of tools and a technological database based on designed operations, have been developed. For their generation DBMS of Delphi - DBE (Borland Database Engine) with a format of type Paradox 7 is used.

\section{CONCLUSION}

A structural diagram of are search and information system for visualization and analysis of errors in making threading tools has been designed.

A main algorithm of its operation has been created.

A model is selected and a structural diagram of the database system is developed.

Algorithms have been developed and programs for 3 modules have been generated using methods of design and technological design known in the practice.

Designed modules have been tested with a specific tool.
The visualization module allows to simulate the process of making taps and to receive four types of errors in it.

\section{REFERENCES:}

[1] Kartunov S. Computer Aided Design of Technological Processes and Control Programs for Small-Series Production Parts, Gabrovo, Thesis, 1992

[2] Kartunov S., P. Rachev, Computer Integrated Manufacturing (CIM), Gabrovo, printing facilities of TU Gabrovo, 1997, ISBN 954-683-057-7

[3] Angelova D., Kartunov S. Analysis of Technological Errors in Making Thread-Cutting Tools, Sozopol, ADP, 2014, ISBN 13103946, p. 237

[4] Rachev P., Analysis of technological errors in machining cylindrical gears with straight teeth. Gabrovo, Thesis, 2009

\footnotetext{
About the authors

Prof. Dr-Ing. Stefan Kartunov, TU Gabrovo, Bulgaria, Hadji Dimitar Str. 4, Department MU, Mechanical Engineering, Lab. Micro- and Nanotechnologies

Research interests: Technological process of micro- and nano elements, CAD/CAM/CAE-studies, simulations and animations before/in the production

$\mathrm{PhD}$ Student Ing. Detelina Angelova, Technical University, Gabrovo, Bulgaria, Hadji Dimitar Str. 4, Department MU, Mechanical Engineering,
} 